\title{
Affine credit risk models under incomplete information
}

\author{
Rüdiger Frey ${ }^{1}$, Cecilia Prosdocimi ${ }^{2}$, \\ Wolfgang J. Runggaldier ${ }^{2}$ \\ ${ }^{1}$ Department of Mathematics, University of Leipzig 04081 Leipzig, Germany \\ (ruediger.frey@math.uni-leipzig.de). \\ ${ }^{2}$ Dipartimento di Matematica Pura ed Aplicata, Universitá di Padova, \\ Via Trieste 63, 35121 Padova, Italy \\ (cecilia.prosdocimi@gmail.com, runggal@math.unipd.it).
}

We consider the problem of computing some basic quantities such as defaultable bond prices and survival probabilities in a credit risk model according to the intensity based approach. We let the default intensities depend on an external factor process that we assume is not observable. We use stochastic filtering to successively update its distribution on the basis of the observed default history. On one hand this allows us to capture aspects of default contagion (information-induced contagion). On the other hand it allows us to evaluate the above quantities also in our incomplete information context. We consider in particular affine credit risk models and show that in such models the nonlinear filter can be computed via a recursive procedure. This then leads to an explicit expression for the filter that depends on a finite number of sufficient statistics of the observed interarrival times for the defaults provided one chooses an initial distribution for the factor process that is of the Gamma type.

Key words: Credit risk, affine models, incomplete information, pricing of credit derivatives, nonlinear filtering, finite dimensional filters.

\section{Introduction}

We consider the problem of evaluating some basic quantities such as defaultable bond prices and survival probabilities in a credit risky environment under incomplete information on the underlying model. We use the reduced-form or intensity-based approach to credit risk with default times 
modeled as the jump times of a doubly stochastic Poisson process. In this model class default intensities are driven by a common factor process $X_{t}$; this is a convenient way for generating dependence between default events of different firms. Typically it is assumed that $X_{t}$ is not directly observable, and this will also be the main setting here. In that case the distribution of $X_{t}$ can be updated on the basis of the observed default history and this leads to what may be termed as information-induced contagion (see e.g. [8]).

In the next section 2 we discuss our underlying model and describe three examples of basic quantities in a credit risky environment (prices of defaultable bonds with and without recovery and survival probabilities) that we first evaluate under the assumption of full knowledge also of the factor process with the main purpose of motivating the filtering problem that arises in the evaluation of these same quantities when only the defaults are observable, but not the values of $X_{t}$. In section 3 we discuss the filtering problem and present its general solution. Although in explicit form, this general solution will in most cases be difficult to compute and so the interest arises to consider particular classes of models, for which the solution can actually be computed. One such class corresponds to the case when $X_{t}$ is a finite-state Markov chain and this is discussed in [4] in a more general context. In this paper we shall concentrate on the so-called affine case and this is the subject of section 4 , where we show that the filter can be computed via a recursive algorithm. The actual computation of this recursive algorithm is discussed in the last section 5, where we also show that for a suitable choice of the initial distribution of the factor process the filter can be computed as an explicit function of a finite number of sufficient statistics of the observed default interarrival times.

In this paper we assume that the information available to an agent comes only from observing the default history. More general information structures can be envisaged such as in the case when agents can observe also prices of defaultable bonds. This generalization is considered in [4], but not for the affine case.

\section{The model and some basic examples}

Consider a market with $m$ firms that may default and denote by $\tau_{j}$ the default time of firm $j \in\{1, \cdots, m\}$. The default state of the portfolio can be summarized by the default indicator process

$$
\mathbf{Y}_{t}=\left(Y_{t, 1}, \cdots, Y_{t, m}\right)_{t \geq 0} \quad \text { with } \quad Y_{t, j}=1_{\left\{\left\{\tau_{j} \leq t\right\}\right\}}
$$

Given a filtered probability space $\left(\Omega, \mathcal{F}, \mathcal{G}_{t}, P\right)$, all processes will be $\mathcal{G}_{t}$-adapted and $\tau_{j}$ is an $\mathcal{G}_{t}$-stopping time. Our intensity-based default model implies that there are no common defaults among the firms, so that 
we may introduce the ordered default times $0=T_{0}<T_{1}<\cdots<T_{m}$. One may then also consider what can be called the default-identity process $\xi_{n}$ that denotes the identity of the firm defaulting at $T_{n}$. The default observation history $\mathcal{H}_{t} \subset \mathcal{G}_{t}$ can then be given the following two equivalent representations

$$
\mathcal{H}_{t}=\sigma\left\{\mathbf{Y}_{s} ; s \leq t\right\}=\sigma\left\{\left(T_{n}, \xi_{n}\right) ; T_{n} \leq t\right\}
$$

The factor process $\mathbf{X}_{t} \in \mathbb{R}$ may be any Markov process (a specific such process will be considered below, see (6)). We assume that default times are conditionally independent, doubly stochastic random times (see [7], Section 9.6); the default intensity of firm $j$ at time $t$ is given by $\lambda_{j}\left(\mathbf{X}_{t}\right)$ for some function $\lambda_{j}: \mathbb{R} \rightarrow(0, \infty)$. Formally, this means that default times are independent given $\mathcal{F}_{\infty}^{X}=\sigma\left(X_{t}: t \geq 0\right)$ with conditional survival probabilities given by

$$
P\left(\tau_{j}>t \mid \mathcal{F}_{\infty}^{X}\right)=\exp \left(-\int_{0}^{t} \lambda_{j}\left(\mathbf{X}_{s}\right) d s\right) .
$$

\subsection{The affine case}

We shall say that we are in the affine case if $\mathbf{X}_{t}$ satisfies a diffusion equation

$$
d \mathbf{X}_{t}=\mu\left(\mathbf{X}_{t}\right) d t+\sigma\left(\mathbf{X}_{t}\right) d w_{t}
$$

with

$$
\left\{\begin{array}{l}
\mu\left(X_{t}\right)=\alpha X_{t}+\beta \\
\sigma^{2}\left(X_{t}\right)=\gamma X_{t}+\delta
\end{array}\right.
$$

Furthermore, assuming for sake of generality that also the short rate is driven by the factor process, i.e. $r_{t}=r\left(X_{t}\right)$,

$$
\begin{cases}\lambda_{j}\left(X_{t}\right)=\lambda_{j} X_{t}, & \lambda_{j}>0 \\ r\left(X_{t}\right)=r X_{t}, & r>0\end{cases}
$$

In particular, for the process $X_{t}$ we shall consider a Cox-Ingersoll-Ross (CIR)-type model, i.e.

$$
d X_{t}=\left(a-b X_{t}\right) d t+\sigma \sqrt{X_{t}} d w_{t}
$$

with $a, b, \sigma>0$ and $a \geq \frac{\sigma^{2}}{2}$ so that $X_{t}>0$ a.s. which, by (5), will then also imply that $\lambda_{j}\left(X_{t}\right)>0, r\left(X_{t}\right)>0$ a.s. For this affine case in what follows 
we shall be able to derive explicit expressions both in the case of full as well as of partial information. To this effect we recall here the following proposition, which in its general form can e.g. be found in [6], section 6.2.2 by making the following identifications : $t=T-t, \lambda=\beta, \mu=\bar{\lambda}, \psi(T-t)=$ $B(t, T),-a \phi(T-t)=A(t, T)$. The particular case for $\beta=0$ can also be found in [7], section 9.5.2. The derivation is based on the Kolmogorov equation for functionals of Markov processes.

Proposition 1. Let $X_{t}$ satisfy (6) and define

$$
F(t, x):=E_{t, x}\left\{e^{-\beta X_{t}} \exp \left[-\int_{t}^{T} \bar{\lambda} X_{s} d s\right]\right\}
$$

for a generic $\beta \geq 0$ and $\bar{\lambda}>0$. In the present affine case this function $F(t, x)$ admits the representation

$$
F(t, x)=\exp [A(t, T)-B(t, T) x]
$$

where, for given $T$, the functions $A(\cdot, T), B(\cdot, T)$ satisfy the following first order ordinary differential equations in $t \in[0, T]$

$$
\begin{cases}B_{t}(t, T)=b B(t, T)+\frac{1}{2} \sigma^{2} B^{2}(t, T)-\bar{\lambda}, & B(T, T)=\beta \\ A_{t}(t, T)=B(t, T) & , A(T, T)=0\end{cases}
$$

and they have as solutions

$$
\left\{\begin{array}{l}
B(t, T)=\frac{\beta\left[\gamma+b+e^{\gamma(T-t)}(\gamma-b)\right]+2 \bar{\lambda}\left(e^{\gamma(T-t)}-1\right)}{\beta \sigma^{2}\left(e^{\gamma(T-t)}-1\right)+\gamma-b+e^{\gamma(T-t)}(\gamma+b)} \\
A(t, T)=\frac{2 a}{\sigma^{2}} \log \left(\frac{2 \gamma e^{\frac{(T-t)(\gamma+b)}{2}}}{\beta \sigma^{2}\left(e^{\gamma(T-t)}-1\right)+\gamma-b+e^{\gamma(T-t)}(\gamma+b)}\right)
\end{array}\right.
$$

with $\gamma:=\sqrt{b^{2}+2 \sigma^{2} \bar{\lambda}}$.

\subsection{Examples}

Of the following three examples the first two concern pricing under full information and so the underlying probability measure $P$ has to be seen as a pricing (martingale) measure. The third one concerns survival probabilities and there $P$ represents then the historical/real world probability measure. The basic quantities in these three examples may be considered as building blocks for more important credit risky products. 
2.2.1 Example 1. Defaultable zero-coupon bond on firm $j$ with maturity $T$ and zero recovery

Using standard results for pricing defaultable claims in models with doubly-stochastic default times (see e.g. [7], section 9.4.3) the price at time $t \leq T$ of a zero recovery bond on firm $j$ can be expressed as

$$
\begin{aligned}
p_{j}(t, T) & =E\left(e^{-\int_{t}^{T} r\left(X_{s}\right) d s}\left(1-Y_{T, j}\right) \mid \mathcal{G}_{t}\right) \\
& =\left(1-Y_{t, j}\right) E_{X_{t}}\left(e^{-\int_{t}^{T} R_{j}\left(X_{s}\right) d s}\right):=\Pi_{1}^{j}\left(X_{t}, \mathbf{Y}_{t}\right)
\end{aligned}
$$

where

$$
R_{j}\left(X_{t}\right):=r\left(X_{t}\right)+\lambda_{j}\left(X_{t}\right)
$$

It is thus a function $\Pi_{1}^{j}\left(X_{t}, \mathbf{Y}_{t}\right)$, parametrized by $t, T$ that for simplicity we drop from the notation, of the current values of the factor and the default indicator processes.

From the previous section 2.1 it is easily seen that in the affine case the function $\Pi_{1}^{j}\left(X_{t}, \mathbf{Y}_{t}\right)$ takes the following exponentially affine form

$$
\Pi_{1}^{j}\left(X_{t}, \mathbf{Y}_{t}\right)=\left(1-Y_{t, j}\right) \exp \left[\alpha^{j}(t, T)-\beta^{j}(t, T) x\right]
$$

where, for $X_{t}$ satisfying the CIR model (6), the coefficients in (13) are given by the formulae in (10) with $\beta^{j}(t, T)$ given by the expression for $B(t, T)$ there and $\alpha^{j}(t, T)$ by that for $A(t, T)$. Furthermore, for the present case the coefficients in the right hand side of (10) have to be chosen as follows (we may consider $t, T$ as fixed): $a, b, \sigma$ come from (6), $\beta=0, \bar{\lambda}=\lambda_{j}+r ; \gamma=$ $\sqrt{b^{2}+2 \sigma^{2} \bar{\lambda}}$.

\subsubsection{Example 2. Recovery payment}

Denote by $Z_{\tau_{j}}^{j} 1_{\left\{\tau_{j} \leq T\right\}}$ the recovery payment at the time $\tau_{j}$ of default of the $j$-th firm, where $Z_{t}^{j}$ is an $\mathcal{F}_{t}^{X}$ - adapted process. It is well-known that the value in $t$ of the recovery payment is given by

$$
\begin{aligned}
& \left(1-Y_{t, j}\right) E\left(e^{-\int_{t}^{\tau_{j}} r\left(\mathbf{X}_{s}\right) d s} Z_{\tau_{j}}^{j} 1_{\left\{\tau_{j} \leq T\right\}} \mid \mathcal{G}_{t}\right) \\
& \quad=\left(1-Y_{t, j}\right) E_{X_{t}}\left(\int_{t}^{T} Z_{s}^{j} \lambda_{j}\left(X_{s}\right) e^{-\int_{t}^{s} R\left(X_{u}\right) d u} d s\right):=\Pi_{2}^{j}\left(X_{t}, \mathbf{Y}_{t}\right) ;
\end{aligned}
$$

see again [7] for a proof.

From these building blocks the price of many credit derivatives is obtained in a straightforward manner. For instance, the price of a zero-coupon 
bond with recovery is simply given by the sum of the price without recovery and the value of the recovery payment, and also spreads of credit default swaps are easily computed. In the affine case also $\Pi_{2}^{j}\left(X_{t}, \mathbf{Y}_{t}\right)$ can be given a more explicit form that is partly of the exponentially affine type. We do not discuss this in detail here referring the reader to [7], section 9.5.3 or directly to the original paper [3].

\subsubsection{Example 3. Survival probabilities}

As already mentioned, in this example the underlying probability $P$ is the historical/real world probability measure. We want in fact to compute the probability, given our information, that firm $j$ does not default prior to a given time $T$. A similar argument as in the derivation of (11) immediately gives

$$
P\left(\tau_{j}>T \mid \mathcal{G}_{t}\right)=\left(1-Y_{t, j}\right) E_{X_{t}}\left\{\exp \left[-\int_{t}^{T} \lambda_{j}\left(X_{s}\right) d s\right]\right\}:=\Pi_{3}^{j}\left(X_{t}, \mathbf{Y}_{t}\right)
$$

Notice that the expression of $\Pi_{3}^{j}\left(X_{t}, \mathbf{Y}_{t}\right)$ is completely analogous to that of $\Pi_{1}^{j}\left(X_{t}, \mathbf{Y}_{t}\right)$ in (11) so that in the affine case it can be given an expression of the exponentially affine form like $\Pi_{1}^{j}\left(X_{t}, \mathbf{Y}_{t}\right)$ in (13).

\section{Incomplete information (the filtering problem)}

In the examples of the previous section we have seen that, under full information also of the factor process $X_{t}$, the values of the basic quantities of interest can be expresses as an explicit function $\Pi\left(X_{t}, \mathbf{Y}_{t}\right)$ of the current values of the factor and the default indicator processes. If agents do not have access to the full information represented by the filtration $\mathcal{G}_{t}$, but only to that corresponding to $\mathcal{H}_{t}$, i.e. the information represented by the default history then, based on iterated conditional expectations, it appears natural to consider as corresponding values for the basic quantities the following

$$
\Pi_{t}\left(\mathbf{Y}_{t}\right):=E\left\{\Pi\left(X_{t}, \mathbf{Y}_{t}\right) \mid \mathcal{H}_{t}\right\}
$$

where the expectation is under the measure $P$ that is a pricing (martingale) measure in the case of the first two examples and the historical measure in the third. For the third example there is no problem with the definition (16), but in the case of the pricing examples one has to make sure that (16) leads to arbitrage-free prices. To this effect we can state the following simple lemma

Lemma 2. Assume the short rate is $\mathcal{H}_{t}$-adapted, in particular deterministic. Then, taking as numeraire the money-market account $\mathcal{B}_{t}:=\exp \left[\int_{0}^{t} r_{s} d s\right]$, formula (16) leads to arbitrage-free prices in the sense that $\mathcal{B}_{t}^{-1} \Pi_{t}\left(\mathbf{Y}_{t}\right)$ is a $\left(P, \mathcal{H}_{t}\right)$-martingale. 
Proof : Let $s \leq t$; then

$$
\begin{aligned}
& E\left\{\frac{\Pi_{t}\left(Y_{t}\right)}{\mathcal{B}_{t}} \mid \mathcal{H}_{s}\right\}=E\left\{E\left\{\frac{\Pi\left(X_{t}, \boldsymbol{Y}_{t}\right)}{\mathcal{B}_{t}} \mid \mathcal{H}_{t}\right\} \mid \mathcal{H}_{s}\right\} \\
& =E\left\{\frac{\Pi\left(X_{t}, Y_{t}\right)}{\mathcal{B}_{t}} \mid \mathcal{H}_{s}\right\}=E\left\{E\left\{\frac{\Pi\left(X_{t}, Y_{t}\right)}{\mathcal{B}_{t}} \mid \mathcal{G}_{s}\right\} \mid \mathcal{H}_{s}\right\} \\
& =E\left\{\frac{\Pi\left(X_{s}, Y_{s}\right)}{\mathcal{B}_{s}} \mid \mathcal{H}_{s}\right\}=\frac{\Pi_{s}\left(Y_{s}\right)}{\mathcal{B}_{s}}
\end{aligned}
$$

where we have used the fact that $P$ is a pricing (martingale) measure for the numeraire $\mathcal{B}_{t}$ in the sense that $\mathcal{B}_{t}^{-1} \Pi\left(X_{t}, \mathbf{Y}_{t}\right)$ is a $P$-martingale in the full filtration $\mathcal{G}_{t}$.

\subsection{The filtering problem}

We have seen that the problem of computing values of risk-sensitive products under incomplete information about the factor process amounts to that of computing conditional expectations as in (16). Since $\mathbf{Y}_{t} \in \mathcal{H}_{t}$, in what follows we shall for simplicity drop the dependence on $\mathbf{Y}_{t}$ so that the right hand side of (16) becomes of the form $E\left\{f\left(X_{t}\right) \mid \mathcal{H}_{t}\right\}$ where $f(\cdot)$ is a generic (bounded) function of the factor process. Denoting by $\pi_{t}(d x)$ the conditional distribution of $X_{t}$ given $\mathcal{H}_{t}$, (16) leads then to the problem of computing

$$
\pi_{t}(f):=E\left\{f\left(X_{t}\right) \mid \mathcal{H}_{t}\right\}=\int f(x) \pi_{t}(d x)
$$

which is a nonlinear filtering problem of a diffusion process, given point process observations.

\subsection{General solution of the filtering problem}

Let us first introduce the global default intensity

$$
\bar{\lambda}\left(X_{t}, \mathbf{Y}_{t}\right):=\sum_{j=1}^{m}\left(1-Y_{t, j}\right) \lambda_{j}\left(X_{t}\right)
$$

which is the sum of the default intensities of the still surviving firms. Note that, for $T_{n} \leq t<T_{n+1}, \bar{\lambda}\left(X_{t}, \mathbf{Y}_{t}\right)$ is the intensity of $T_{n+1}$.

In deriving our filtering results we distinguish the cases between default times and at a default time.

\subsubsection{Filter between defaults}

Let $T_{n}$ be the generic $n$-th default time and let $t \in\left[T_{n}, T_{n+1}\right)$. It follows from the general filtering equations (Kushner-Sratonovich equations) for point process observations by the so-called innovations method (see [5], [2]) that for $\pi_{t}(f)$ as defined in (17) one has

$$
\pi_{t}(f)=\pi_{T_{n}}(f)+\int_{T_{n}}^{t}\left[\pi_{s}(\mathcal{L} f)-\pi_{s}(\bar{\lambda} f)+\pi_{s}(\bar{\lambda}) \pi_{s}(f)\right] d s
$$


where $\mathcal{L}$ is the generator that corresponds to the diffusion process $X_{t}$. Furthermore, noticing that for $t \in\left[T_{n}, T_{n+1}\right)$ one has $\mathbf{Y}_{t}=\mathbf{Y}_{T_{n}}$, whenever $t \in\left[T_{n}, T_{n+1}\right)$ we shall consider $\bar{\lambda}$ as a function of $x$ alone, i.e.

$$
\bar{\lambda}(x)=\bar{\lambda}\left(x, \mathbf{Y}_{T_{n}}\right)
$$

with $\bar{\lambda}(x, \mathbf{y})$ as in (18).

Proposition 3. Assume the conditions for the uniqueness of the solution of (19) as described e.g. in Appendix 2 of [2] hold. The solution to (19) is then for $t \in\left[T_{n}, T_{n+1}\right.$ ) and an integrable (bounded) $f$ given by

$$
\pi_{t}(f)=\frac{\varrho_{t}(f)}{\varrho_{t}(1)}
$$

where $\varrho_{t}(f)$, the unnormalized conditional expectation of $f$, can be obtained as

$$
\varrho_{t}(f)=\int \psi_{t}\left(T_{n}, x\right)(f) \pi_{T_{n}}(d x)
$$

with

$$
\psi_{t}\left(T_{n}, x\right)(f):=E_{T_{n}, x}\left\{f\left(t, X_{t}\right) e^{-\int_{T_{n}}^{t} \bar{\lambda}\left(X_{s}\right) d s}\right\}
$$

and $\bar{\lambda}\left(X_{t}\right)$ according to (20).

Remark 4. This proposition shows that, between defaults, the filter evolves deterministically and its evolution is determined by the Markovian semigroup $\psi_{t}\left(T_{n}, x\right)(f)$ in $(23)$.

Proof: Although the proof can be obtained from that of Proposition 3.4 in [2], we present here a direct derivation.

By Ito's formula we have

$$
\begin{aligned}
& f\left(t, X_{t}\right) e^{-\int_{T_{n}}^{t} \bar{\lambda}\left(X_{s}\right) d s}-f\left(T_{n}, X_{T_{n}}\right) \\
& \quad=\int_{T_{n}}^{t} e^{-\int_{T_{n}}^{s} \bar{\lambda}\left(X_{u}\right) d u}\left[(\mathcal{L} f)\left(s, X_{s}\right)-\bar{\lambda}\left(X_{s}\right) f\left(s, X_{s}\right)\right] d s \\
& \quad+\int_{T_{n}}^{t} e^{-\int_{T_{n}}^{s} \bar{\lambda}\left(X_{u}\right) d u} \sigma \sqrt{X_{s}} \frac{\partial f}{\partial x}\left(s, X_{s}\right) d w_{s}
\end{aligned}
$$

Assuming the conditions for applying Fubini's theorem are satisfied, let us take on the left and right hand sides the expectation conditional on $X_{T_{n}}=x$ 
thus obtaining

$$
\begin{aligned}
& E_{T_{n}, x}\left\{f\left(t, X_{t}\right) e^{-\int_{T_{n}}^{t} \bar{\lambda}\left(X_{s}\right) d s}\right\}-f\left(T_{n}, x\right) \\
& \quad=\int_{T_{n}}^{t} E_{T_{n}, x}\left\{e^{-\int_{T_{n}}^{s} \bar{\lambda}\left(X_{u}\right) d u}\left[(\mathcal{L} f)\left(s, X_{s}\right)-\bar{\lambda}\left(X_{s}\right) f\left(s, X_{s}\right)\right] d s\right\}
\end{aligned}
$$

Using the definition of $\psi_{t}$ in (23) this last relation can be rewritten as

$$
\psi_{t}\left(T_{n}, x\right)(f)-\psi_{T_{n}}\left(T_{n}, x\right)(f)=\int_{T_{n}}^{t}\left[\psi_{s}\left(T_{n}, x\right)(\mathcal{L} f)-\psi_{s}\left(T_{n}, x\right)(\bar{\lambda} f)\right] d s
$$

Integrating with respect to $\pi_{T_{n}}(d x)$, taking into account (22) and applying once more Fubini, one arrives at

$$
\varrho_{t}(f)-\varrho_{T_{n}}(f)=\int_{T_{n}}^{t}\left[\varrho_{s}(\mathcal{L} f)-\varrho_{s}(\bar{\lambda} f)\right] d s
$$

From here one obtains then immediately

$$
d \pi_{t}(f)=d\left(\frac{\varrho_{t}(f)}{\varrho_{t}(1)}\right)=\pi_{t}(\mathcal{L} f) d t-\pi_{t}(\bar{\lambda} f) d t+\pi_{t}(f) \pi_{t}(\bar{\lambda}) d t
$$

from which the result follows by the assumed uniqueness of the solution of (19).

\subsubsection{Filter at a default}

Consider now a generic default time $T_{n}$. Again from the general filtering equations of the innovations approach ([5],[2]) one has

$$
\pi_{T_{n}}(f)=\frac{\pi_{T_{n}^{-}}\left(\lambda_{\xi_{n}} f\right)}{\pi_{T_{n}^{-}}\left(\lambda_{\xi_{n}}\right)}
$$

where we implicitly use that $\pi_{T_{n}^{-}}\left(\lambda_{\xi_{n}}\right)>0$ a.s. The expression $\pi_{T_{n}^{-}}(f)$ denotes here the left hand limit of $\pi_{T_{n}}(f)$ in $T_{n}$, which exists by (19).

Concluding this section 3.2 one sees that the crucial point to obtain a solution of the filtering equations is the possibility of explicitly computing the semigroup $\psi_{t}$ in (23) and in the next Section 4 we shall address this issue in the case of an affine model.

\section{Filtering in affine models}

As mentioned at the end of the previous section, in this section we shall derive an explicit representation of the semigroup $\psi_{t}$ in (23) for affine models and this will then lead to an explicit solution of the filtering problem. 
Recall that for an affine model we have postulated an affine dynamics for $X_{t}$ that we take here as given by the CIR model (6). Furthermore, as in (5), we shall assume $\lambda_{j}\left(X_{t}\right)=\lambda_{j} X_{t}$, which by (18) and (20) then implies that also $\bar{\lambda}\left(X_{t}, \mathbf{Y}_{t}\right)=\bar{\lambda}\left(\mathbf{Y}_{t}\right) \cdot X_{t}$; for the short rate we assume that it is constant, i.e. $r\left(X_{t}\right) \equiv r$. Finally, we shall assume $f(t, x)$ to be exponentially affine, analogously to $F(t, x)$ in (8), namely of the form

$$
f(t, x)=\exp [\alpha(t, T)-\beta(t, T) x]
$$

and its specific form depends on the specific problem at hand (see the examples in section 2.2). Notice next that with $f(t, x)$ of the form (25) the semigroup $\psi_{t}$ becomes

$$
\begin{aligned}
\psi_{t}\left(T_{n}, x\right)(f) & =E_{T_{n}, x}\left\{e^{\alpha(t, T)-\beta(t, T) X_{t}} e^{-\bar{\lambda} \int_{T_{n}}^{t} X_{s} d s}\right\} \\
& =e^{\alpha(t, T)} E_{T_{n}, x}\left\{e^{-\beta(t, T) X_{t}} e^{-\bar{\lambda} \int_{T_{n}}^{t} X_{s} d s}\right\}
\end{aligned}
$$

where we have simply written $\bar{\lambda}$ for $\bar{\lambda}\left(\mathbf{Y}_{t}\right)$ since $\mathbf{Y}_{t}=\mathbf{Y}_{T_{n}}$. The crucial quantity becomes therefore the second factor in the rightmost expression in (26). We have now the following proposition, the proof of which follows immediately from Proposition 1

Proposition 5. Under the assumptions of this section one has

$$
E_{T_{n}, x}\left\{e^{-\beta(t, T) X_{t}} e^{-\bar{\lambda} \int_{T_{n}}^{t} X_{s} d s}\right\}=\exp \left[A\left(T_{n}, t ; \beta\right)-B\left(T_{n}, t ; \beta\right) x\right]_{\mid \beta=\beta(t, T)}
$$

where $A\left(T_{n}, t ; \beta\right)$ and $B\left(T_{n}, t ; \beta\right)$ are given by

$$
\left\{\begin{array}{l}
A\left(T_{n}, t ; \beta\right)=\frac{2 a}{\sigma^{2}} \log \left(\frac{2 \gamma e^{\frac{\left(t-T_{n}\right)(\gamma+b)}{2}}}{\beta \sigma^{2}\left(e^{\gamma\left(t-T_{n}\right)}-1\right)+\gamma-b+e^{\gamma\left(t-T_{n}\right)}(\gamma+b)}\right) \\
B\left(T_{n}, t ; \beta\right)=\frac{\beta\left[\gamma+b+e^{\gamma\left(t-T_{n}\right)}(\gamma-b)\right]+2 \bar{\lambda}\left(e^{\gamma\left(t-T_{n}\right)}-1\right)}{\beta \sigma^{2}\left(e^{\gamma\left(t-T_{n}\right)}-1\right)+\gamma-b+e^{\gamma\left(t-T_{n}\right)}(\gamma+b)}
\end{array}\right.
$$

with $\gamma:=\sqrt{b^{2}+2 \sigma^{2} \bar{\lambda}}$

\subsection{Filter between defaults}

Combining Propositions 3 and 5 and noticing that the constant 1 can be expressed as the function $f(t, x)$ in (25) for $\alpha(t, T)=\beta(t, T)=0$, one immediately obtains 
Proposition 6. The filter $\pi_{t}(f)$ is, for $t \in\left[T_{n}, T_{n+1}\right)$, given by

$$
\pi_{t}(f)=\eta\left(T_{n} ; t, T\right) \frac{\int e^{-B\left(T_{n}, t ; \beta(t, T)\right) x} \pi_{T_{n}}(d x)}{\int e^{-B\left(T_{n}, t ; 0\right) x} \pi_{T_{n}}(d x)}
$$

where

$$
\eta\left(T_{n} ; t, T\right)=e^{\alpha(t, T)} \exp \left[A\left(T_{n}, t ; \beta(t, T)\right)-A\left(T_{n}, t ; 0\right)\right]
$$

Remark 7. It follows from (28) that $B\left(T_{n}, t ; \beta\right)$ is, for $t \geq T_{n}$, nonnegative whenever $\beta \geq \bar{\beta}(t)$ where

$$
\bar{\beta}(t)=-\min \left[\frac{2 \bar{\lambda}\left(e^{\gamma\left(t-T_{n}\right)}-1\right)}{\gamma+b+e^{\gamma\left(t-T_{n}\right)}(\gamma-b)}, \frac{\gamma-b+e^{\gamma\left(t-T_{n}\right)}(\gamma+b)}{\sigma^{2}\left(e^{\gamma\left(t-T_{n}\right)}-1\right)}\right]
$$

Thus $\bar{\beta}(t)$ is strictly positive for $t>T_{n}$ and it is equal to zero for $t=T_{n}$. From the examples in section 2.2 it follows that the value of $\beta(t, T)$ to be used for the parameter $\beta$ in the numerator of the right hand side in (29) (as well as in (34) below) is strictly positive, while in the denominator of the same formulae it is equal to zero. Consequently the corresponding value of $B\left(T_{n}, t ; \beta\right)$ is always nonnegative. In (34) below we shall also need the derivative of $B\left(T_{n}, t ; \beta\right)$ with respect to $\beta$, evaluated at $\beta=\beta\left(T_{n+1}, T\right)$ and at $\beta=0$. Since $T_{n+1}>T_{n}$ and thus $\bar{\beta}\left(T_{n+1}\right)<0$, not only $\beta=\beta\left(T_{n+1}, T\right)$, but also $\beta=0$ are interior points of the domain of positivity for $B\left(T_{n}, t ; \beta\right)$ and in this domain it is easily seen from (28) that $B\left(T_{n}, t ; \beta\right)$ is differentiable with respect to $\beta$. Recall now also that $X_{t}$ as given by (6) for $a \geq \frac{\sigma^{2}}{2}$ has its a-priori support in the positive half line and this implies that also all conditional distributions $\pi_{t}(d x)$ have their support in the positive half line. It follows that the two integrals in the right hand side of (29) are well defined and finite.

From the above Remark 7 we have that, for all values of interest for $\beta$, $B\left(T_{n}, t ; \beta\right)$ is strictly positive. Given that $X_{t}>0$ anyway, this leads us to define the moment generating function of the conditional distribution $\pi_{t}(d x)$ of $X_{t}$ by

$$
\chi_{t}(\phi):=\pi_{t}\left(e^{-\phi X_{t}}\right) \quad \phi>0
$$

From Proposition 6 one then obtains immediately

Corollary 8. For $t \in\left[T_{n}, T_{n+1}\right)$ and $f(t, x)$ as in (25) we have that the filter value $\pi_{t}(f)$ is given by

$$
\pi_{t}(f)=\eta\left(T_{n} ; t, T\right) \frac{\chi_{T_{n}}\left(B\left(T_{n}, t ; \beta(t, T)\right)\right.}{\chi_{T_{n}}\left(B\left(T_{n}, t ; 0\right)\right)}
$$

where $\eta(\cdot)$ is as in (30), $\chi_{T_{n}}(\cdot)$ as in (32) and $B\left(T_{n}, t ; \beta\right)$ is given by (28). 


\subsection{Filter at a default time}

Based on the general formula (24) for the filter at a generic default time $T_{n}$, we can now show the following (since for the case between defaults we considered the interval $\left[T_{n}, T_{n+1}\right)$, here we take $T_{n+1}$ to denote the generic default time)

Proposition 9. Assuming the conditions are fulfilled to differentiate under the integral sign, at the generic default time $T_{n+1}$ we have

$$
\pi_{T_{n+1}}(f)=e^{\alpha\left(T_{n+1}, T\right)} \cdot \frac{\frac{\partial}{\partial \beta}\left[e^{A\left(T_{n}, T_{n+1} ; \beta\right)} \chi_{T_{n}}\left(B\left(T_{n}, T_{n+1} ; \beta\right)\right)\right]_{\mid \beta=\beta\left(T_{n+1}, T\right)}}{\frac{\partial}{\partial \beta}\left[e^{A\left(T_{n}, T_{n+1} ; \beta\right)} \chi_{T_{n}}\left(B\left(T_{n}, T_{n+1} ; \beta\right)\right)\right]_{\mid \beta=0}}
$$

where $A\left(T_{n}, T_{n+1} ; \beta\right)$ and $B\left(T_{n}, T_{n+1} ; \beta\right)$ are given by $(28), \beta\left(T_{n+1}, T\right)$ corresponds to the exponentially affine representation of $f(t, x)$ in $(25)$ and $\chi_{T_{n}}(\cdot)$ is as defined in (32).

Proof: Starting from (24) for the default time $T_{n+1}$ and noticing that $\pi_{T_{n+1}^{-}}(f)$ is the limit, for $t \uparrow T_{n+1}$, of the filter $\pi_{t}(f)$ when $t \in\left[T_{n}, T_{n+1}\right)$, using Proposition 3 with $f(t, x)$ as in (25) and with the semigroup $\psi_{t}(\cdot)$ as in (26) combined with Proposition 5, we obtain the following sequence of equalities, where we use differentiation under the integral sign in two instances

$$
\begin{aligned}
& \pi_{T_{n+1}}(f)=\frac{\pi_{T_{n+1}^{-}}\left\{\lambda_{\xi_{n+1}} \cdot X_{T_{n+1}} f\left(T_{n+1}, X_{T_{n+1}}\right)\right\}}{\pi_{T_{n+1}^{-}}\left\{\lambda_{\xi_{n+1}} \cdot X_{T_{n+1}}\right\}} \\
& =\frac{e^{\alpha\left(T_{n+1}, T\right)} \int E_{T_{n}, x}\left\{X_{T_{n+1}} e^{-\beta\left(T_{n+1}, T\right) \cdot X_{T_{n+1}}} e^{-\bar{\lambda} \int_{T_{n}}^{T_{n+1}} X_{u} d u}\right\} \pi_{T_{n}}(d x)}{\left.\int E_{T_{n}, x}\left\{X_{T_{n+1}} e^{-\bar{\lambda} \int_{T_{n}}^{T_{n+1}} X_{u} d u}\right\}\right\} \pi_{T_{n}}(d x)} \\
& =e^{\alpha\left(T_{n+1}, T\right)} \frac{\left.\int \frac{\partial}{\partial \beta} E_{T_{n}, x}\left\{e^{\beta X_{T_{n+1}}} e^{-\bar{\lambda} \int_{T_{n}}^{T_{n+1}} X_{u} d u}\right\}\right\}_{\mid \beta=-\beta\left(T_{n+1}, T\right)} \pi_{T_{n}}(d x)}{\int \frac{\partial}{\partial \beta} E_{T_{n}, x}\left\{e^{\beta X_{T_{n+1}}} e^{-\bar{\lambda} \int_{T_{n}}^{T_{n+1}} X_{u} d u}\right\}_{\mid \beta=0} \pi_{T_{n}}(d x)} \\
& =e^{\alpha\left(T_{n+1}, T\right)} \frac{\int \frac{\partial}{\partial \beta}\left\{\exp \left[A\left(T_{n}, T_{n+1} ; \beta\right)-B\left(T_{n}, T_{n+1} ; \beta\right) x\right]\right\}_{\mid \beta \beta \beta\left(T_{n+1}, T\right)} \pi_{T_{n}}(d x)}{\int \frac{\partial}{\partial \beta}\left\{\exp \left[A\left(T_{n}, T_{n+1} ; \beta\right)-B\left(T_{n}, T_{n+1} ; \beta\right) x\right]\right\}_{\mid \beta=0} \pi_{T_{n}}(d x)} \\
& =e^{\alpha\left(T_{n+1}, T\right)} \frac{\frac{\partial}{\partial \beta}\left[e^{A\left(T_{n}, T_{n+1} ; \beta\right)} \chi_{T_{n}}\left(B\left(T_{n}, T_{n+1} ; \beta\right)\right)\right]_{\beta=\beta\left(T_{n+1}, T\right)}}{\frac{\partial}{\partial \beta}\left[e^{A\left(T_{n}, T_{n+1} ; \beta\right)} \chi_{T_{n}}\left(B\left(T_{n}, T_{n+1} ; \beta\right)\right)\right]_{\beta=0}}
\end{aligned}
$$

Notice that, by the considerations in Remark 7 all the above quantities are well defined. 
Remark 10. Notice that in the filter update at $T_{n+1}$ only the information about the timing $T_{n+1}$ of the $(n+1)$-st default is being used and not also that of the defaulting firm $\xi_{n+1}$; in fact, the factor $\lambda_{\xi_{n+1}}$, which contains this information, drops out due to the normalization. This happens however only because of our affine form of the dependence of the default intensity on the factor process and the fact that the latter is taken to be scalar. In any case, the information about $\xi_{n+1}$ is not lost as it becomes part of $\mathbf{Y}_{T_{n+1}}$.

From Corollary 8 and Proposition 9 it now follows that the crucial quantity for computing the filter in the affine case is the moment generating function $\chi_{t}(\phi)$ in (32), which we need to compute only for the values of $t$ corresponding to the default times and for any $\phi>0$. Notice also that $\chi_{t}(\phi)$ is nothing but the filter $\pi_{t}(f)$ for $f(t, x)$ when this latter function has the exponentially affine form in (25) with $\alpha(t, T)=0$ and $\beta(t, T)=\phi$. Combining these remarks with the results of Proposition 9 one obtains immediately

Corollary 11. Under the assumptions of Proposition 9 one has

$$
\chi_{T_{n+1}}(\phi)=\frac{\frac{\partial}{\partial \beta}\left[e^{A\left(T_{n}, T_{n+1} ; \beta\right)} \chi_{T_{n}}\left(B\left(T_{n}, T_{n+1} ; \beta\right)\right)\right]_{\mid \beta=\phi}}{\frac{\partial}{\partial \beta}\left[e^{A\left(T_{n}, T_{n+1} ; \beta\right)} \chi_{T_{n}}\left(B\left(T_{n}, T_{n+1} ; \beta\right)\right)\right]_{\mid \beta=0}}
$$

On the basis of the previous results we can now write down an algorithm to compute recursively the filter in the affine case, which we do in the next subsection.

\subsection{Filter algorithm}

i) Start at $T_{0}=0$ with a given $\chi_{0}(\phi)$.

ii) At the generic $T_{n+1}$ compute (see Corollary 11)

$$
\chi_{T_{n+1}}(\phi)=\frac{\frac{\partial}{\partial \beta}\left[e^{A\left(T_{n}, T_{n+1} ; \beta\right)} \chi_{T_{n}}\left(B\left(T_{n}, T_{n+1} ; \beta\right)\right)\right]_{\mid \beta=\phi}}{\frac{\partial}{\partial \beta}\left[e^{A\left(T_{n}, T_{n+1} ; \beta\right)} \chi_{T_{n}}\left(B\left(T_{n}, T_{n+1} ; \beta\right)\right)\right]_{\mid \beta=0}}
$$

iii) For $t \in\left[T_{n}, T_{n+1}\right)$ and with $f(t, x)=\exp [\alpha(t, T)-\beta(t, T) x]$ the filter is then given by (see Corollary 8 )

$$
\pi_{t}(f)=\eta\left(T_{n} ; t, T\right) \frac{\chi_{T_{n}}\left(B\left(T_{n}, t ; \beta(t, T)\right)\right.}{\chi_{T_{n}}\left(B\left(T_{n}, t ; 0\right)\right)}
$$

where $\eta\left(T_{n} ; t, T\right)$ is given in (30) and $B\left(T_{n}, t ; \beta\right)$ in (28).

iv) For $t=T_{n+1}$ the filter is (see Proposition 9 and Corollary 11)

$$
\pi_{T_{n+1}}(f)=e^{\alpha\left(T_{n+1}, T\right)} \chi_{T_{n+1}}\left(\beta\left(T_{n+1}, T\right)\right)
$$


Step ii) above is a recursive formula to compute the moment generating function $\chi_{T_{n}}$ that then leads to an explicit filter solution according to Steps iii) and iv). Although recursive in nature, this Step ii) may become increasingly difficult to compute since the analytic expression for $\chi_{T_{n}}(\phi)$ might become more and more involved with every step. A natural question then arises to see whether, with a suitable choice of the initial $\chi_{0}(\phi)$, the recursions remain at a level that allows for feasible computations. This will be the subject of the next Section 5, where we also show that, although a priori the filter is not finite-dimensional in the traditional sense, for a suitable choice of the initial distribution it can be parametrized by a finite number of sufficient statistics.

Remark 12. We conclude this section by pointing out that our filter results may have a wider scope than only what we have described here by showing that the filter can be computed by computing the conditional moment generating function and that this can be done recursively according to (35). In fact, since this conditional moment generating function is related to the Laplace transform of the conditional (filter) distribution, one can, at least in theory, invert this conditional moment generating function thereby recovering the filter distribution itself. This would then allow to compute conditional expectations not only of exponentially affine functions of the factor process but also of any integrable function thereof.

\section{Finite dimensional computation of the filter}

In this section we exhibit a choice for $\chi_{0}(\phi)$ that allows Step ii) in the filter algorithm of the previous subsection 4.3 to remain computable at every step.

For this purpose recall that the recursions in Step ii) correspond to the recursive formula (35), where the coefficients $A\left(T_{n}, T_{n+1} ; \beta\right), B\left(T_{n}, T_{n+1} ; \beta\right)$ are given in (28). Introduce the shorthand notations

$$
\left\{\begin{array}{l}
R_{n+1}=\gamma+b+e^{\gamma\left(T_{n+1}-T_{n}\right)}(\gamma-b) \\
S_{n+1}=2 \bar{\lambda}\left(e^{\gamma\left(T_{n+1}-T_{n}\right)}-1\right) \\
U_{n+1}=\sigma^{2}\left(e^{\gamma\left(T_{n+1}-T_{n}\right)}-1\right) \\
V_{n+1}=\gamma-b+e^{\gamma\left(T_{n+1}-T_{n}\right)}(\gamma+b) \\
W_{n+1}=2 \gamma e^{\frac{\left(T_{n+1}-T_{n}\right)(\gamma+b)}{2}}
\end{array}\right.
$$

that, since $\gamma=\sqrt{b^{2}+2 \sigma^{2}} \bar{\lambda}$, are all positive quantities. The coefficients $A\left(T_{n}, T_{n+1} ; \beta\right)$ and $B\left(T_{n}, T_{n+1} ; \beta\right)$ from (28) can then be given the following 
representation

$$
\left\{\begin{array}{l}
A\left(T_{n}, T_{n+1} ; \beta\right)=\frac{2 a}{\sigma^{2}} \log \left(\frac{W_{n+1}}{\beta U_{n+1}+V_{n+1}}\right) \\
B\left(T_{n}, T_{n+1} ; \beta\right)=\frac{\beta R_{n+1}+S_{n+1}}{\beta U_{n+1}+V_{n+1}}
\end{array}\right.
$$

Choosing as distribution for the initial value $X_{0}$ of the factor process a specific Gamma-type distribution, we shall now prove the following theorem that gives an explicit computable representation for $\chi_{t}(\phi)$ at the various default times $t=T_{n}$.

Theorem 13. Let

$$
\chi_{0}(\phi)=\left(\frac{1}{1+\phi}\right)^{\frac{2 a}{\sigma^{2}}}=(1+\phi)^{-\frac{2 a}{\sigma^{2}}}, \quad \phi>0
$$

which according to (32) corresponds to the moment generating function of a Gamma distribution for $X_{0}$ with parameters $\left(\frac{2 a}{\sigma^{2}}, 1\right)$. Then

$$
\chi_{T_{n}}(\phi)=c_{n}\left(\phi H_{n}+K_{n}\right)^{-\frac{2 a}{\sigma^{2}}-n} p_{n}(\phi)
$$

where $H_{n}$ and $K_{n}$ satisfy the recursions

$$
\begin{cases}H_{n}=R_{n} H_{n-1}+U_{n} K_{n-1} & , \quad H_{0}=1 \\ K_{n}=S_{n} H_{n-1}+V_{n} K_{n-1} & , \quad K_{0}=1\end{cases}
$$

the coefficient $c_{n}$ is given by

$$
c_{n}=\left[K_{n}^{-\frac{2 n}{\sigma^{2}}-n} p_{n}(0)\right]^{-1}
$$

and $p_{n}(\phi)$ is a polynomial of degree $n-1$ given by

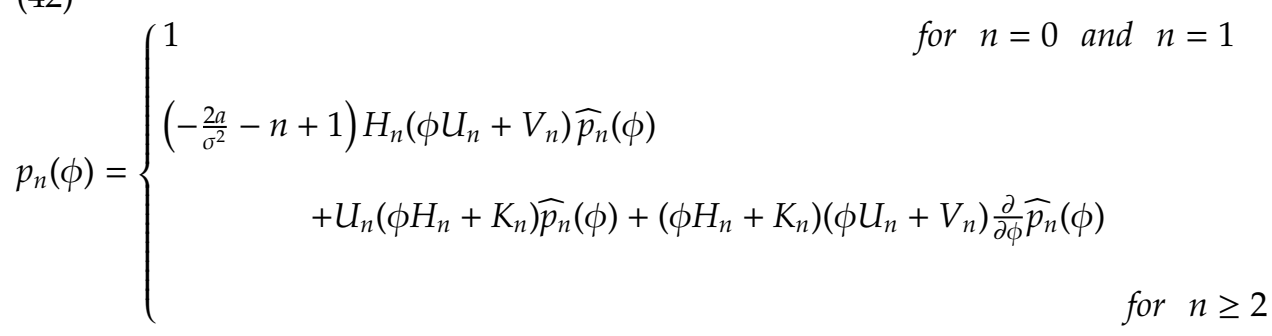

with

$$
\widehat{p}_{n}(\phi)=\left(\phi U_{n}+V_{n}\right)^{n-2} p_{n-1}\left(\frac{\phi R_{n}+S_{n}}{\phi U_{n}+V_{n}}\right), \quad n \geq 2
$$


Proof: The statement is clearly true for $n=0$. We show it first for $n=1$ and then inductively for all $n \geq 2$.

i) the case $n=1$ : by (35),(36), (37) and the recursions in (40) we have

$(44)$

$$
\chi_{T_{1}}(\phi)=\frac{\frac{\partial}{\partial \beta}\left\{\left(\frac{W_{1}}{\beta U_{1}+V_{1}}\right)^{\frac{2 a}{\sigma^{2}}} \chi_{T_{0}}\left(\frac{\beta R_{1}+S_{1}}{\beta U_{1}+V_{1}}\right)\right\}_{\beta \beta=\phi}}{\frac{\partial}{\partial \beta}\left\{\left(\frac{W_{1}}{\beta U_{1}+V_{1}}\right)^{\frac{2 a}{\sigma^{2}}} \chi_{T_{0}}\left(\frac{\beta R_{1}+S_{1}}{\beta U_{1}+V_{1}}\right)\right\}_{\beta \beta=0}}
$$

$$
=\frac{\frac{\partial}{\partial \beta}\left\{\left(\frac{W_{1}}{\beta U_{1}+V_{1}}\right)^{\frac{2 a}{\sigma^{2}}}\left(\frac{\beta H_{1}+K_{1}}{\beta U_{1}+V_{1}}\right)^{-\frac{2 a}{\sigma^{2}}}\right\}_{\mid \beta=\phi}}{\frac{\partial}{\partial \beta}\left\{\left(\frac{W_{1}}{\beta U_{1}+V_{1}}\right)^{\frac{2 a}{\sigma^{2}}}\left(\frac{\beta H_{1}+K_{1}}{\beta u_{1}+V_{1}}\right)^{-\frac{2 a}{\sigma^{2}}}\right\}_{\mid \beta=0}}=\frac{\left(\phi H_{1}+K_{1}\right)^{-\frac{2 a}{\sigma^{2}}-1}}{K_{1}^{-\frac{2 a}{\sigma^{2}}-1}}
$$

which indeed corresponds to (39) with (41) and (42).

ii) the general case $n \geq 2$ : assume (39) holds for $n-1$. Then, always by (35), (36), (37) and (40) we obtain

$$
\begin{aligned}
& \chi_{T_{n}}(\phi)=\frac{\frac{\partial}{\partial \beta}\left\{\left(\frac{W_{n}}{\beta U_{n}+V_{n}}\right)^{\frac{2 a}{\sigma^{2}}} \chi T_{n-1}\left(\frac{\beta R_{n}+S_{n}}{\beta U_{n}+V_{n}}\right)\right\}_{\mid \beta=\phi}}{\frac{\partial}{\partial \beta}\left\{\left(\frac{W_{n}}{\beta U_{n}+V_{n}}\right) \frac{2 a}{\sigma^{2}} \chi \chi_{T_{n-1}}\left(\frac{\beta R_{n}+S_{n}}{\beta U_{n}+V_{n}}\right)\right\}_{\mid \beta=0}} \\
& =\frac{\frac{\partial}{\partial \beta}\left\{\left(\frac{1}{\beta u_{n}+V_{n}}\right)^{\frac{2 a}{\sigma^{2}}}\left(\frac{\beta H_{n}+K_{n}}{\beta u_{n}+V_{n}}\right)^{\frac{2 a}{\sigma^{2}}-n+1} p_{n-1}\left(\frac{\beta R_{n}+S_{n}}{\beta U_{n}+V_{n}}\right)\right\}_{\beta=\phi}}{\frac{\partial}{\partial \beta}\left\{\left(\frac{1}{\beta U_{n}+V_{n}}\right)^{\frac{2 a}{2}}\left(\frac{\beta H_{n}+K_{n}}{\beta u_{n}+V_{n}}\right)^{-\frac{2 a}{\sigma^{2}}-n+1} p_{n-1}\left(\frac{\beta R_{n}+S_{n}}{\beta U_{n}+V_{n}}\right)\right\}_{\beta=0}}
\end{aligned}
$$

Taking into account that, by (43),

$$
p_{n-1}\left(\frac{\beta R_{n}+S_{n}}{\beta U_{n}+V_{n}}\right)=\left(\beta U_{n}+V_{n}\right)^{2-n} \widehat{p}_{n}(\beta)
$$

the numerator in the rightmost expression of (45) becomes

$$
\begin{aligned}
& \frac{\partial}{\partial \beta}\left\{\left(\beta U_{n}+V_{n}\right)\left(\beta H_{n}+K_{n}\right)^{-\frac{2 a}{\sigma^{2}}-n+1} \widehat{p}_{n}(\beta)\right\}_{\mid \beta=\phi} \\
& =\left(\beta H_{n}+K_{n}\right)^{-\frac{2 n}{\sigma^{2}}-n}\left\{U_{n}\left(\beta H_{n}+K_{n}\right) \widehat{p}_{n}(\beta)\right. \\
& \left.+\left(-\frac{2 a}{\sigma^{2}}-n+1\right) H_{n}\left(\beta U_{n}+V_{n}\right) \widehat{p}_{n}(\beta)+\left(\beta U_{n}+V_{n}\right)\left(\beta H_{n}+K_{n}\right) \frac{\partial}{\partial \beta} \widehat{p}_{n}(\beta)\right\}_{\mid \beta=\phi} \\
& =\left(\phi H_{n}+K_{n}\right)^{-\frac{2 a}{\sigma^{2}}-n} p_{n}(\phi)
\end{aligned}
$$


where we have used the definition of $p_{n}(\phi)$ in (42). Returning to (45) and recalling that the denominator in the rightmost expression of (45) is the same as the numerator except for putting $\beta=0$, one finally obtains

$$
\chi_{T_{n}}(\phi)=\frac{\left(\phi H_{n}+K_{n}\right)^{-\frac{2 n}{\sigma^{2}}-n} p_{n}(\phi)}{K_{n}^{-\frac{2 n}{\sigma^{2}}-n} p_{n}(0)}=c_{n}\left(\phi H_{n}+K_{n}\right)^{-\frac{2 a}{\sigma^{2}}-n} p_{n}(\phi)
$$

Remark 14. It follows from Theorem 13 that, for a choice of the initial distribution corresponding to $\chi_{0}(\phi)$ in (38), the sequence $\chi_{T_{n}}(\phi)$ and therefore (see Steps iii) and iv) in Section 4.3) the entire filter is parameterized by a same finite number of sufficient statistics, namely the pairs $\left(H_{n}, K_{n}\right)$ and the polynomial functions $p_{n}(\phi)$, all of which can be computed recursively on the basis of the functions $R_{n}, S_{n}, U_{n}, V_{n}$ of the interarrival times of the defaults.

\section{References}

1. Bielecki, T. R., M. Jeanblanc, \& M. Rutkowski (2004). Modeling and Valuation of Credit Risk. In : Stochastic Methods in Finance (M. Frittelli and W. Runggaldier, eds), Lecture Notes in Mathematics. Vol 1856, 27-126. Springer Verlag.

2. Ceci,C., \& A. Gerardi (2006). A Model for High Frequency Data under Partial Information : a Filtering Approach. International Journal of Theoretical and Applied Finance (to appear).

3. Duffie, D., \& N. Gârleanu (2001). Risk and Valuation of Collateralized Debt Obligations. Financial Analysts Journal. 57, 41-59.

4. Frey, R., \& W. J. Runggaldier ( 2006). Credit Risk and Incomplete Information : a Nonlinear Filtering Approach. Preprint.

5. Kliemann,W. H., G. Koch, \& F. Marchetti (1990). On the Unnormalized Solution of the Filtering Problem with Counting Process Observations. IEEE Transactions on Information Theory. 36, 1415-1425.

6. Lamberton,D., \& B. Lapeyre (1995). An Introduction to Stochastic Calculus Applied to Finance. Chapman and Hall.

7. McNeil A. J., R. Frey, \& P. Embrechts (2005). Quantitative Risk Management. Princeton University Press.

8. Schönbucher, P. (2004) Information-driven Default Contagion. Preprint, Department of Mathematics, ETH Zürich. 